\title{
Influência do tabagismo na força muscular respiratória em idosos
}

\author{
Influence of smoking on respiratory muscle strength in elderly \\ Eliane Regina Ferreira Sernache de Freitas', Evelize Cristina Labegaline da Silva Araujo², Katia da Silva Alves ${ }^{3}$
}

RESUMO I Este estudo consistiu em avaliar a influência do tabagismo na força muscular respiratória (pressões inspiratória máxima e expiratória máxima) em idosos fisicamente independentes, comparando-os àqueles não fumantes. Foram selecionados 120 idosos com idades iguais ou acima de 60 anos de ambos os sexos e divididos em dois grupos de acordo com os critérios da Organização Mundial de Saúde: Grupo Tabagistas, com 14, e Grupo Não tabagistas, com 106 indivíduos. Foi aplicado um questionário estruturado para coleta de dados demográficos. Para avaliação da força muscular respiratória, utilizou-se o manovacuômetro analógico da marca Gerar ${ }^{\oplus}$, com intervalo de -300 a +300 $\mathrm{cmH}_{2} \mathrm{O}$. Foram utilizados o teste $t$ de Student para avaliar a diferença entre as médias encontradas e o coeficiente de correlação de Pearson ( $\left.r^{2}\right)$ para analisar as associações. O Grupo Tabagistas apresentou menor pressão inspiratória máxima com diferença significante $(p<0,0001)$. Não houve diferença significativa na pressão expiratória máxima. Observou-se que o índice de massa corpórea também foi significantemente inferior nos fumantes ( $p<0,0001)$, porém não foi identificada correlação positiva com força muscular inspiratória. Esses resultados sugerem que o tabagismo está relacionado com a redução da força da musculatura inspiratória e que, apesar de não apresentar correlação positiva com esta diminuição de força muscular, o índice de massa corpórea também foi menor em tais indivíduos.

Descritores I idoso; força muscular; respiração; tabagismo.

\begin{abstract}
I This study evaluated the influence of smoking on respiratory muscle strength (maximal inspiratory and maximal expiratory pressures) in physically independent elderly compared to nonsmokers. We selected 120 aged people equal or above 60 yearsold divided into two groups, according to the World Health Organization criteria: Smokers Group, including 106 individuals, and Nonsmokers Group, with 14. A structured questionnaire was applied to collect demographic data. For respiratory muscle strength evaluation, a GERAR ${ }^{\oplus}$ analog manovacuometer with a range of -300 to $+300 \mathrm{cmH}_{2} \mathrm{O}$ was used. Student's $t$-test was used to evaluate the difference between means, and Pearson's correlation coefficient $\left(r^{2}\right)$ to anaIyze associations. The Smokers Group presented lower maximum inspiratory pressure with a significant difference $(p<0.0001)$. There was no significant difference in the maximum expiratory pressure. It was observed that the body mass index was also significantly lower in smokers ( $p<0.0001$ ), however no positive correlation with inspiratory muscle strength was found. These results suggest that smoking is related with reduction of the inspiratory muscle strength, and that although it does not present a positive correlation with such decreased muscle strength, body mass index was also reduced in such individuals.
\end{abstract}

Keywords I aged; muscle strength; respiration; smoking.

Estudo desenvolvido no Centro de Pesquisa em Ciências da Saúde da Universidade Norte do Paraná (CPCS-UNOPAR) - Londrina (PR), Brasil. 'PhD; Professora do Departamento de Fisioterapia da UNOPAR - Londrina (PR), Brasil. 2MSc; Professora do Departamento de Fisioterapia da UNOPAR - Londrina (PR), Brasil.

${ }^{3}$ Professora do Departamento de Fisioterapia da UNOPAR - Londrina (PR), Brasil. 


\section{INTRODUÇÃO}

O processo de envelhecimento provoca alterações no sistema respiratório, tais como a redução da mobilidade da caixa torácica e da elasticidade pulmonar e diminuição dos valores da pressão inspiratória (PImáx) e expiratória máximas (PEmáx), com consequente redução da eficiência de tosse, bem como diminuição da mobilidade dos cílios do epitélio respiratório ${ }^{1}$. Outra característica associada ao processo de envelhecimento é o declínio da função muscular respiratória, o que provoca hipoventilação, baixa tolerância ao exercício e dispneia ${ }^{2}$.

A atrofia da musculatura esquelética, como ocorrência fisiológica do envelhecimento, provoca maior diminuição de sua força e potência muscular, sendo relatada na literatura como um fato relacionado diretamente à idade 3 . Esse processo, denominado sarcopenia, geralmente prevalece na população idosa e possivelmente ocorre tanto na musculatura periférica quanto na respiratória ${ }^{4}$.

A força muscular respiratória (FMR) pode ser mensurada por meio das pressões sub e supra-atmosférica que tais músculos são capazes de gerar, sendo, portanto, a PImáx, a maior que pode ser gerada durante a inspiração forçada contra uma via aérea ocluída; e a PEmáx a maior que pode ser desenvolvida durante um vigoroso esforço expiratório contra uma via aérea ocluída ${ }^{5}$.

Diferenças nas características da musculatura esquelética entre fumantes e não fumantes têm sido demonstradas em alguns estudos ${ }^{6,7}$. A porcentagem e o diâmetro das fibras tipo I encontram-se inferiores nos indivíduos fumantes ${ }^{6}$. Além disso, a capacidade oxidativa dos músculos esqueléticos de indivíduos fumantes é menor, a julgar pelo decréscimo na atividade enzimática mitocondrial ${ }^{6}$. No entanto, ainda não está claro na literatura se a força dos músculos respiratórios sofre a influência do tabagismo.

Sendo assim, o objetivo do presente estudo foi avaliar a influência do tabagismo em idosos fisicamente independentes, na FMR (PImáx e PEmáx), comparativamente aos não fumantes.

\section{METODOLOGIA}

O estudo se caracterizou por uma investigação do tipo transversal, quantitativa e observacional, seguindo os critérios estabelecidos pela Strenghthening the Reporting of Observacional Studies in Epidemioly (STROBE) ${ }^{8}$. O trabalho foi aprovado pelo Comitê de Ética e Pesquisa da Universidade Norte do Paraná (CEP/UNOPAR) 0034/09. Todos os indivíduos que aceitaram participar do estudo assinaram o termo de consentimento livre e esclarecido.

A escolha da amostra processou-se de maio de 2009 a junho de 2010. Foi constituída inicialmente por 138 idosos com idades iguais ou acima de 60 anos, fisicamente independentes, cadastrados nas Unidades Básicas de Saúde (UBS) da zona urbana de Londrina, no Paraná, Brasil, e integrantes do projeto "Estudo sobre Envelhecimento e Longevidade"(EELO), desenvolvido pela Universidade Norte do Paraná

A amostragem foi definida de forma aleatória estratificada, levando-se em consideração o gênero das quatro regiões do município de Londrina e totalizando 13 UBS, sendo: quatro da região Norte, quatro da Sul, duas da Leste e três da Oeste.

\section{Protocolo do estudo}

Os sujeitos foram sorteados e contatados por telefone ou por visitas domiciliares por meio de suas respectivas UBS e responderam ao questionário de autoavaliação da capacidade funcional. O objetivo desta etapa foi classificar os idosos em fisicamente independentes de acordo com os níveis três e quatro do estado funcional, proposto por Spirduso 9 e com ausência de distúrbio cognitivo evidenciado por meio do Miniexame do Estado Mental (MEEM) ${ }^{10}$. Foram excluídos os idosos que informaram cirurgia torácica ou abdominal recente, apresentaram dor torácica de origem cirúrgica, doença pulmonar aguda relatada e doença neurológica que pudesse prejudicar a realização e/ou dificultar o entendimento para execução dos testes.

Posteriormente, os idosos incluídos no estudo responderam a um questionário para avaliação do hábito de fumar, definindo-se como fumante, de acordo com os critérios da Organização Mundial de Saúde (OMS) ${ }^{11}$, todo indivíduo que, por ocasião do inquérito, estivesse fumando qualquer tipo ou quantidade de tabaco, diariamente há pelo menos seis meses e como não fumantes, aqueles que nunca tenham fumado ou tenham feito isso por pouco tempo ou de forma esporádica, qualquer tipo ou quantidade de tabaco. O grau de dependência do tabaco foi avaliado pelo questionário de tolerância de Fagerström ${ }^{12}$. O objetivo de tal questionário é identificar e medir a dependência nicotínica conforme a soma de pontos obtidos com as respostas referentes às seis perguntas (Quadro 1), classificando a dependência nicotínica em cinco graus: muito baixa (0 a 2), baixa (3 a 4), 
Quadro 1 - Perguntas do questionário de tolerância de Fagerströn

\begin{tabular}{|c|c|c|}
\hline \multirow[t]{5}{*}{1} & \multicolumn{2}{|l|}{$\begin{array}{l}\text { Pergunta 1: Quanto tempo após acordar você fuma o seu } \\
\text { primeiro cigarro? }\end{array}$} \\
\hline & Dentro de 5 minutos & 3 \\
\hline & Entre 6 e 30 minutos & 2 \\
\hline & Entre 31 e 60 minutos & 1 \\
\hline & Após 60 minutos & O \\
\hline 2 & $\begin{array}{l}\text { Pergunta 2: Você acha difícil não fumar em locais onde o } \\
\text { fumo é proibido (como igrejas, biblioteca etc.)? }\end{array}$ & \\
\hline & $\operatorname{Sim}$ & 1 \\
\hline & Não & O \\
\hline 3 & $\begin{array}{l}\text { Pergunta 3: Qual o cigarro do dia traz mais satisfação (ou que } \\
\text { mais detestaria deixar de fumar)? }\end{array}$ & \\
\hline & O primeiro da manhã & 1 \\
\hline & Outros & O \\
\hline 4 & Pergunta 4: Quantos cigarros você fuma por dia? & \\
\hline & 10 ou menos & O \\
\hline & 11 a 20 & 1 \\
\hline & 21 a 31 & 2 \\
\hline & 31 ou mais & 3 \\
\hline 5 & $\begin{array}{l}\text { Pergunta 5: Você fuma mais frequentemente pela manhã (nas } \\
\text { primeiras horas do dia) que no resto do dia? }\end{array}$ & \\
\hline & Sim & 1 \\
\hline & Não & O \\
\hline 6 & $\begin{array}{l}\text { Pergunta 6: Você fuma mesmo quando está tão doente que } \\
\text { precisa ficar de cama a maior parte do tempo? }\end{array}$ & \\
\hline & $\operatorname{Sim}$ & 1 \\
\hline & Não & 0 \\
\hline
\end{tabular}

média (5), elevada (6 a 7) e muito elevada (8 a 10). Para o ponto de corte entre fumantes com baixa e elevada dependência do tabaco, adotou-se a mediana, cujo valor foi seis. Assim, os fumantes que somaram acima de seis pontos (dependência elevada ou muito elevada) foram considerados como: elevada dependência de nicotina (EDN), e aqueles com menos de seis pontos (dependência média, baixa ou muito baixa) foram considerados: baixa dependência de nicotina (BDN). Todos os participantes foram submetidos à avaliação do peso e da altura e, a seguir, à avaliação da FMR.

\section{Avaliação da força muscular respiratória}

Para mensuração da força dos músculos respiratórios por meio da medida da PImáx e da PEmáx, utilizou-se manovacuômetro analógico da marca GERAR ${ }^{\circledR}$ com escala de $-300 \mathrm{a}+300 \mathrm{cmH}_{2} \mathrm{O}$. As medições foram realizadas com o paciente na posição sentada confortavelmente, pés apoiados, mãos sobre as coxas, com clip nasal e utilizando bocal descartável. O manovacuômetro foi conectado ao bocal por meio de uma cânula, havendo entre esta e o instrumento uma conexão com orifício de $1 \mathrm{~mm}$ de diâmetro para evitar a influência da musculatura orofacial nos valores pressóricos ${ }^{13}$.
Para medida da PImáx, o paciente foi orientado a exalar até o volume residual, a partir do qual deveria realizar um esforço inspiratório máximo. Para medida da PEmáx, o paciente devia inspirar o mais profundamente possível até a capacidade pulmonar total, em seguida, efetuar um esforço expiratório máximo até o volume residual. Ambas as manobras deveriam ser mantidas com esforço de pressão máxima por, no mínimo, dois segundos, com o orifício do tubo ocluído ${ }^{13}$.

Realizaram-se no mínimo três medidas aceitáveis e reprodutíveis, isto é, sem vazamento e com diferença inferior a 10\%, intercaladas com dois minutos de repouso. Entre as mensurações da PImáx e da PEmáx, houve um intervalo de cinco minutos ${ }^{14}$. Dos três valores obtidos, tanto na PImáx quanto na PEmáx, apenas o maior valor foi considerado ${ }^{14}$. As medidas foram feitas por um único examinador. $\mathrm{O}$ maior valor obtido para cada indivíduo e considerado na análise foi comparado com aquele esperado, de acordo com a equação de $\mathrm{Neder}^{5}$ proposta para população brasileira, sendo expressos em percentagem (\%PImáx e \%PEmáx).

\section{Análise estatística}

A análise dos dados coletados foi realizada separando as amostras em dois grupos: tabagistas e não tabagistas. $\mathrm{Na}$ comparação entre as variáveis que preencheram os critérios de normalidade, adotou-se o teste $t$ de Student para avaliar a diferença de médias encontradas. $\mathrm{O}$ coeficiente de correlação de Pearson $\left(r^{2}\right)$ foi utilizado para avaliar as associações entre força muscular, sexo e índice de massa corpórea (IMC) em relação ao tabagismo. $\mathrm{O}$ nível de significância adotado foi de $5 \%(\mathrm{p}<0,05)$. O software utilizado para análise estatística foi o SPSS, versão 16.0 (Chicago, IL, USA).

\section{RESULTADOS}

Foram recrutados inicialmente 138 idosos, entretanto, 18 deles foram excluídos: oito não compreenderam e/ou conseguiram realizar a manobra para realização do teste, seis apresentaram tosse no momento das manobras e quatro tiveram queixa de dor torácica durante a realização do teste. Desta forma, foram avaliados 120 idosos que preencheram os critérios de inclusão e conseguiram realizar os testes (39,2\% homens; $60,8 \%$ mulheres).

A média e o desvio padrão das idades dos participantes foram de $68,4 \pm 6,5$ anos, variando de 60 a 89 , 
e o IMC foi de 27,5 $\pm 4,4$, variando de 17,5 a 36,6 . Em relação ao tabagismo, $11,7 \%$ dos idosos eram fumantes (GT, n=14) e 88,3\% não fumantes (GNT, n=106), como observado na Tabela 1.

Foram realizadas em média 3,9 0 ,92 manobras para medida da PImáx e da PEmáx, variando entre três a seis manobras. Pela análise dos resultados, verificou-se que existe alteração das medidas da FMR em idosos tabagistas quando comparados aos não tabagistas, principalmente na \%PImáx, a qual apresentou uma significante redução de seus valores no Grupo GT $(p<0,01)$. Em relação \%PEmáx, apesar de não demonstrar significância estatística, observou-se uma discreta redução em seus valores quando comparado aos não fumantes $\left(\mathrm{GT}=90,3 \pm 34,7 \quad \mathrm{cmH}_{2} \mathrm{O} ; \mathrm{GNT}=97,3 \pm 30,6 \mathrm{cmH}_{2} \mathrm{O}\right)$, como visto na Tabela 2 .

\begin{tabular}{|c|c|c|c|}
\hline Variáveis & $\begin{array}{c}\mathrm{GT} \\
\mathrm{n}=14(11,7 \%)\end{array}$ & $\begin{array}{c}\text { GNT } \\
\mathrm{n}=106(88,3 \%)\end{array}$ & Valor $\mathrm{p}$ \\
\hline Idade (anos) & $69,0 \pm 6,6$ & $64,6 \pm 3,7$ & \\
\hline \multicolumn{4}{|l|}{ Sexo - n (\%) } \\
\hline Masculino & $06(42,9)$ & $12(11,3)$ & \multirow{2}{*}{0,640} \\
\hline Feminino & $08(57,1)$ & $21(19,8)$ & \\
\hline IMC & $23,5 \pm 4,9$ & $28,0 \pm 4,1$ & 0,000 \\
\hline PImáx (medido) & $90,6 \pm 27,9$ & $62,5 \pm 22,0$ & 0,000 \\
\hline PEmáx (medido) & $102,0 \pm 31,5$ & $90,0 \pm 34,3$ & 0,186 \\
\hline \multicolumn{4}{|l|}{$\begin{array}{l}\text { Dependência de } \\
\text { nicotina - n (\%) }\end{array}$} \\
\hline EDN & $04(28,6)$ & & \multirow{2}{*}{ 0,301 } \\
\hline BDN & $10(71,4)$ & & \\
\hline MEEM & $25,8 \pm 4,0$ & $26,1 \pm 4,1$ & 0,661 \\
\hline
\end{tabular}

GT: Grupo Tabagista; GNT: Grupo Não tabagista; PImáx: pressão inspiratória máxima; PEmáx: pressão expiratória máxima: IMC: índice de massa corpórea: EDN: elevada dependência de nicotina; BDN: baixa dependência de nicotina; MEEM: miniexame do estado mental

Tabela 2. Comparação das pressões respiratórias de acordo com o hábito de fumar

\begin{tabular}{|c|c|c|c|}
\hline Variáveis & $\begin{array}{c}\text { GT } \\
n=14(11,7 \%)\end{array}$ & $\begin{array}{c}\text { GNT } \\
\mathrm{n}=106(88,3 \%)\end{array}$ & Valor $p$ \\
\hline PImáx (\% do predito) & $63,9 \pm 29,3$ & $97,9 \pm 30,6$ & 0,000 \\
\hline PEmáx (\% do predito) & $90,3 \pm 34,7$ & $97,3 \pm 30,6$ & 0,431 \\
\hline IMC & $23,5 \pm 4,9$ & $28,0 \pm 4,1$ & 0,000 \\
\hline
\end{tabular}

GT: Grupo Tabagista; GNT: Grupo Não tabagista; PImáx: pressão inspiratória máxima; PEmáx: pressão expiratória máxima; IMC: índice de massa corpórea.

Tabela 3. Comparação das pressões respiratórias entre os sexos masculino e feminino

\begin{tabular}{lcccc}
\multirow{2}{*}{$\begin{array}{l}\text { Hábito } \\
\text { de fumar }\end{array}$} & \multicolumn{1}{c}{$\begin{array}{c}\text { Pressões } \\
\text { respiratórias }\end{array}$} & $\begin{array}{c}\text { Masculino } \\
\mathrm{n}=44(39,2 \%)\end{array}$ & $\begin{array}{c}\text { Feminino } \\
\mathrm{n}=73(60,8 \%)\end{array}$ & Valor p \\
GT & Plmáx (\% do predito) & $74,1 \pm 27,4$ & $56,2 \pm 30,0$ & 0,276 \\
& PEmáx (\% do predito) & $75,9 \pm 26,5$ & $76,8 \pm 32,7$ & 0,892 \\
GNT & Plmáx (\% do predito) & $88,8 \pm 23,7$ & $91,34 \pm 42,8$ & 0,896 \\
& PEmáx (\% do predito) & $104,5 \pm 32,7$ & $93,6 \pm 29,0$ & 0,082
\end{tabular}

GT: Grupo Tabagista; GNT: Grupo Não tabagista; PImáx: pressão inspiratória máxima; PEmáx: pressão expiratória máxima.
O IMC também foi significativamente inferior nos idosos fumantes $(\mathrm{p}<0,001)$, entretanto não apresentou correlação com a PImáx $\left(r^{2}=-0,088 ; p=0,063\right)$ ou com a PEmáx $\left(r^{2}=-0,053 ; p=0,236\right)$, vide Tabela 2 .

A Tabela 3 apresenta os dados relativos à análise da \%PImáx e da \%PEmáx de idosos tabagistas e não tabagistas em relação ao sexo, que não demonstrou nenhuma diferença entre os grupos.

\section{DISCUSSÃO}

A prevalência de tabagismo em idosos verificada neste estudo $(11,7 \%)$ foi semelhante à observada no estado de São Paulo ${ }^{15}$; em Porto Alegre, no Rio Grande do Sul (11,6\%) $)^{16}$; na "Pesquisa Especial de Tabagismo" (PeTab, 12,9\% em maiores de 64 anos de idade $)^{17}$ e em um inquérito que envolveu 16 capitais brasileiras $(12,7 \%)^{18}$.

Os resultados do presente estudo mostraram que a força muscular inspiratória (PImáx) encontrava-se reduzida nos idosos fumantes quando comparada à dos não fumantes. Entretanto, a força muscular expiratória (PEmáx) não apresentou diferença entre os grupos. A PImáx é considerada como o índice da força dos músculos inspiratórios, principalmente do músculo diafragma, e a PEmáx refere-se à força daqueles expiratórios, principalmente dos abdominais ${ }^{19}$. Sabe-se que o músculo diafragma é composto em sua maior parte por fibras musculares tipo $\mathrm{I}^{20}$, propiciando contração lenta, alta capacidade oxidativa e baixa capacidade glicolítica, relacionadas com desempenho e resistência aeróbica ${ }^{21}$. Essas particularidades dos músculos respiratórios podem explicar os resultados encontrados, pois importantes achados em relação às características morfológicas, metabólicas e funcionais das fibras musculares esqueléticas em fumantes têm sido relatados, tais como: proporção reduzida de fibras tipos I e IIa, aumento das fibras tipo IIb, reduzida capacidade oxidativa e alta capacidade glicolítica ao considerar o decréscimo nas atividades das enzimas mitocondriais e reduzida fração de fibras e de volume mitocondrial ${ }^{6}$.

Alguns autores concluíram que a função muscular esquelética em fumantes apresenta menor resistência à fadiga muscular e força isométrica e dinâmica ${ }^{22}$. Há também relatos de que a atividade endotelial de óxido nítrico muscular pode estar prejudicada no fumante, entretanto, não há evidências 
que apoiem a presença de inflamação muscular esquelética local ${ }^{23}$.

Estudos demonstraram que a inalação de monóxido de carbono ( $\mathrm{CO})$, resultando em carboxihemoglobina $(\mathrm{COHb})$, foi encontrada em níveis elevados em indivíduos fumantes. Estes indivíduos apresentaram maior fadiga muscular quando comparados aos não fumantes, o que sugeriu um impacto agudo do $\mathrm{CO}$ na habilidade resistiva do músculo esquelético ${ }^{24,25}$.

No presente estudo observou-se também que o IMC de idosos fumantes foi significamente menor aos dos não fumantes. Estudos realizados em cobaias suínos compararam os efeitos sistêmicos da exposição da fumaça do cigarro, constantando que a taxa de ganho de peso corporal foi menor do que no Grupo Controle ${ }^{26,27}$. Para complementar, um dos estudos concluiu que os efeitos da fumaça do cigarro no peso corporal antecedem e parecem ser independente das alterações produzidas nas pequenas vias aére$\mathrm{as}^{27}$. Recentemente, um estudo relacionou indivíduos fumantes com doença pulmonar obstrutiva aos não fumantes e concluiu que os fumantes apresentaram menor composição corporal, incluindo massas gorda e magra e conteúdo mineral ósseo ${ }^{28}$.

O presente estudo apresentou algumas limitações, tais como o não pareamento dos grupos por idade e sexo e a não realização da espirometria, a qual poderia contribuir de forma mais fidedigna na inclusão de idosos, os quais, apesar de serem fumantes, apresentassem função pulmonar normal.

\section{CONCLUSÃO}

Esses resultados sugerem que o tabagismo está relacionado com a redução da força muscular respiratória, em especial da musculatura inspiratória e do IMC. Apesar de não ter apresentado correlação significativa, tais reduções podem sugerir alterações das massas gorda e magra e densidade mineral óssea, podendo precipitar uma maior predisposição à diminuição da capacidade funcional, ao aparecimento de doenças pulmonares e à piora da qualidade de vida. Sendo assim, os resultados do estudo apontam para a necessidade de estratégias à cessação do tabagismo em idosos, com ações específicas para este grupo etário que se apresenta mais vulnerável a doenças pulmonares.

\section{AGRADECIMENTOS}

Para a realização deste estudo, contribuíram idosos integrantes do projeto EELO, aos quais gostaríamos de expressar o nosso agradecimento.

\section{REFERÊNCIAS}

1. Francisco PM, Donalisio MR, de Azevedo Barros MB, Cesar CL, Carandina L, Goldbaum M. [Factors associated with pulmonary disease among the elderly]. Rev Saúde Pública. 2006; 40(3):428-35.

2. Simões PP, Auad MA, Dionísio J, Mazzonetto M. Influência da idade e do sexo na força muscular respiratória. Fisioter Pesqui. 2007:14(1):36-41.

3. Simões RP, Deus APL, Auad MA, Dionísio J, Mazzonetto M, BorghiSilva A. Maximal respiratory pressure in healthy 20 to 89 year-old sedentary individuals of central São Paulo State. Rev Bras Fisioter. 2010;14(1):60-7.

4. Kim J, Sapienza CM. Implications of expiratory muscle strength training for rehabilitation of the elderly: Tutorial. J Rehabil Res Dev. 2005;42(2):211-24

5. Neder JA, Andreoni S, Lerario MC, Nery LE. Reference values for lung function tests. II. Maximal respiratory pressures and voluntary ventilation. Braz J Med Biol Res. 1999;32(6):719-27.

6. Larsson L, Orlander J. Skeletal muscle morphology, metabolism and function in smokers and non-smokers. A study on smoking-discordant monozygous twins. Acta Physiol Scand. 1984;120(3):343-52.

7. Zanoni CT, Rodrigues CMC, Mariano D, Suzan ABBM, Boaventura LC, Galvão F. Efeitos do treinamento muscular inspiratório em universitártios tagabistas e não tabagistas. Inspiratory muscle training effects in smokers and nonsmokers univertitary students. Fisioter Pesqui. 2012;19(2):147-52.

8. Vandenbroucke JP, von EE, Altman DG, Gotzsche PC, Mulrow CD, Pocock SJ, et al. Strengthening the Reporting of Observational Studies in Epidemiology (STROBE): explanation and elaboration. Epidemiology. 2007:18(6):805-35.

9. Spirduso WW. Dimensões físicas do envelhecimento. $1^{\text {a }}$ ed. São Paulo: Manole; 2004.

10. Brucki SM, Nitrini R, Caramelli P, Bertolucci PH, Okamoto $\mathbb{H}$. [Suggestions for utilization of the mini-mental state examination in Brazil]. Arq Neuropsiquiatr. 2003;61(3B):777-81.

11. World Health Organization. Guidelines on working with the private sector to achieve health outcomes-Report by the secretariat [Internet]. Geneva, 2000 (N/WHO/SMO/83 4) 2000 November 30 [cited 2010 Jun 15]. Available from: http://apps.who.int/gb/archive/ pdf_files/EB107/ee2O.pdf

12. Heatherton TF, Kozlowski LT, Frecker RC, Fagerstrom KO. The Fagerstrom Test for Nicotine Dependence: a revision of the Fagerstrom Tolerance Questionnaire. Br J Addict. 1991;86(9):1119-27.

13. Sousa RB. Pressões respiratórias estáticas máximas. J Pneumol. 2002:28(3):155-65.

14. Brunetto AF, Alves LA. Comparing peak and sustained values of maximal respiratory pressures in healthy subjects and chronic pulmonary disease patients. J Pneumol. 2003;29(4):481-5. 
15. Zaitune MPA, Barros MBA, Lima MG, Cesar CLG, Goldbaum M, Alves MCGP. Factors associated with smoking in the elderly: a health survey in São Paulo (ISA-SP). Cad Saúde Pública 2012;28(3):583-95.

16. Paskulin LMG, Vianna LAC. Perfil sociodemográfico e condições de saúde auto-referidas de idosos de Porto Alegre. Rev Saúde Pública. 2007:41(5):757-68

17. Instituto Nacional de Câncer (Brasil). Organização Pan-Americana da Saúde.Rio de Janeiro: INCA. Pesquisa Especial de Tabagismo (PETab) - Relatório Brasil Global Adult Tobacco Survey - Brazil Report Encuesta Global de Tabaquismo en Adultos - Informe Brasil. Rev Bras Cancerol. 2011;57(3):429-30

18. Pereira JC, Barreto SM, Passos VMA. O perfil de saúde cardiovascular dos idosos brasileiros precisa melhorar: estudo de base populacional. Arq Bras Cardiol. 2008;91(1):1-10.

19. Black LF, Hyatt RE. Maximal respiratory pressures: normal values and relationship to age and sex. Am Rev Respir Dis. 1969;99(5):696-702.

20. Celli BR, MacNee W. Standards for the diagnosis and treatment of patients with COPD: a summary of the ATS/ERS position paper. Eur Respir J. 2004:23(6):932-46.

21. Scullion JE. NICE guidelines: the management, treatment and care of COPD. Br J Nurs. 2004;13(18):1100-3.
22. Morse $\mathrm{Cl}$, Wüst RC, Jones DA, de Haan A, Degens H. Muscle fatigue resistance during stimulated contractions is reduced in young male smokers. Acta Physiol (Oxf). 2007;191(2):123-9.

23. Zhang WZ, Venardos K, Chin-Dusting J, Kaye DM. Adverse effects of cigarette smoke on NO bioavailability: role of arginine metabolism and oxidative stress. Hypertension. 2006;48(2):278-85.

24. Wüst RC, Morse $\mathrm{Cl}$, de Haan A, Rittweger J, Jones DA, Degens $\mathrm{H}$. Skeletal muscle properties and fatigue resistance in relation to smoking history. Eur J Appl Physiol. 2008:104(1):103-10.

25. Morse $\mathrm{Cl}$, Pritchard LJ, Wüst RC, Jones DA, Degens $H$. Carbon monoxide inhalation reduces skeletal muscle fatigue resistance. Acta Physiol (Oxf). 2008:192(3):397-401.

26. Ardite E, Peinado VI, Rabinovich RA, Fernandez-Checa JC, Roca $\mathrm{J}$, Barbera JA. Systemic effects of cigarette smoke exposure in the guinea pig. Respir Med. 2006:100(7):1186-94.

27. Castro MRP, Matsuo T, Nunes SOV. Características clínicas e qualidade de vida de fumantes em um centro de referências de abordagem e tratamento do tabagismo. J Bras Pneumol. 2010;36(1):67-74.

28. van den Borst B, Koster A, Yu B, Gosker HR, Meibohm B, Bauer DC, et al. Is age-related decline in lean mass and physical function accelerated by obstructive lung disease or smoking? Thorax. 2011;66(11):961-9. 